\section{Shortcut to protein redesign}

\section{By Michael J. Haas, Senior Writer}

Proteins are desirable therapeutics because of the specificity of their interactions, but wild-type proteins often make suboptimal drugs because of poor bioavailability, pharmacokinetics or other properties. Companies have two main routes to improve these properties: genetic engineering or chemical modification. The latter technique can produce a more diverse range of modifications, but total synthesis and purification of a redesigned protein is lengthy and laborious.

Investigators at the Institute for Research in Biomedicine have been working for the last few years to develop a shortcut to redesigning proteins via chemical modification. In 2002, the researchers reported the development of a combinatorial synthesis technique that could produce redesigned peptides of high purity right on a bead substrate. ${ }^{1}$ Now, the group has added another time-saving component to

"The detection of proteins of
interest is based directly on
molecular recognition. You
are screening directly for the
interaction you are trying to
modify or optimize."
-Ernest Giralt,
Institute for Research in Biomedicine

Eight analogs of the core appeared to replicate the folding properties of the full wild-type domain because they bound IgG. Because the study was proof of concept, the researchers did not seek to optimize the binding. Rather, their goal was to show that altered peptide sequences could yield a domain with similar binding to the original.

Giralt told SciBX that the technique is well suited to optimizing a protein's interactions with other proteins because it offers two advantages over existing approaches.

One is that it is capable of a higher throughput than total synthesis or in vivo methods. "The synthesis of the 300-peptide library, including quality-control analysis, took five days," Giralt told SciBX. "The covalent [total] synthesis of one entire B domain would take about one week, from automatic assembling and cleavage, to purification and final characterization."

"The other advantage is maybe not so evident," he said. "The detection of proteins of interest is based directly on molecular recognition. You are screening directly for the interaction you are trying to modify or optimize," without first having to purify the peptides or cleave them from the beads.

\section{Screening potential}

Although some protein-therapeutics companies contacted by SciBX saw potential for the synthesis technique, they were more interested in the screening technology.

"There could be complementarity between our computational peptide design and Giralt's screening method," said Bassil Dahiyat, presi- the technique: on-bead, high-throughput screening of the modified peptides.

Rather than wanting to adopt the entire technique, biotechs in the protein redesign space told $S c i B X$ that the screening method currently has more potential than the synthesis technique, although both components have limitations.

In the Journal of the American Chemical Society, a group led by Ernest Giralt reported the design of eight analogs of the hydrophobic core of the B domain of staphylococcal protein A. ${ }^{2}$ Giralt is program head at the institute and professor of chemistry at the University of Barcelona.

Giralt's group chose the B domain because its structure and binding properties are well known. Thus, the researchers knew that to alter the $\mathrm{B}$ domain's binding to antibodies, modifications needed to be made to 4 key amino acids found within the 22 -amino-acid hydrophobic core that direct the formation of the domain's surface. At the same time, the entire domain needed to retain its correct folding properties.

Instead of synthesizing the entire B domain and modifying the amino acids, the researchers divided the B domain into two parts-the hydrophobic core and the rest. The group synthesized 300 versions of the hydrophobic core, each analog on a single bead. The beads were then screened against the remaining portion of the B domain and fluorescently tagged IgG. dent and CEO of Xencor Inc. "We could use computational design methods, like our own PDA technology, to prefilter the possible combinations and decide which peptide variations to synthesize. Giralt's approach would provide the experimental screening step."

Xencor uses its Protein Design Automation (PDA) technology to engineer antibodies for cancer and therapeutic proteins for both inflammation and autoimmune disorders. In January, the company began a Phase I trial of XmAb 2513, which targets CD30, for lymphoma and Hodgkin's disease. Xencor has other mAbs targeting CD19 and CD40 in preclinical development for B and T cell lymphomas, leukemia and cancer.

Dahiyat did say that the screening method needs more development before it is ready for commercial applications. "It would be exciting to see it applied to more biologically relevant systems-for example, the redesign of a domain or protein to bind a therapeutically interesting target like a protease or receptor."

For William Boyle, president and CSO of Anaptys Biosciences Inc., a key question is how much throughput the screening technology can handle. "Giralt screened only 300 compounds," he told SciBX. "Could he screen millions of compounds?"

If the answer is yes, Boyle said that Giralt's screening method might prove to be a simpler alternative to methods like protein microarrays, phage display and yeast two-hybrid screening. 


\section{DRUG PLATFORMS}

Giralt's answer is yes. "The screening of the library can be done manually, as in the paper," Giralt said, "or automatically using a dedicated flow cytometer we have available now at our laboratory. Manually one can screen about 5,000 beads per day. Automatically, one can screen 100,000 beads per hour."

Anaptys is using its Omnitope-SHM technology to develop and optimize antibodies against validated, new and previously intractable targets. Somatic hypermutation (SHM) is the natural process by which immune cells adapt to antigens. The company doesn't yet have any disclosed product candidates.

\section{The synthesis side}

Giralt's approach currently cannot produce peptides much larger than about 20 amino acids without compromising purity. Thus, said Dahiyat, Giralt's synthesis method is limited to short peptide sequences.

Dahiyat added that the method "could have an advantage with small peptides or peptidomimetics, because small peptides aren't amenable to recombinant methods. But I think it will have little immediate commercial application in the major areas of protein redesign, for example industrial enzymes, therapeutic proteins and antibodies."

Dahiyat noted that the method "was clever because they used a simple biophysical trick: cutting a protein in two."

Boyle suggested that the approach could address a "major protein engineering task" of inserting non-natural residues into proteins. Currently such proteins must be synthesized in vivo. "Giralt's method could allow site-directed insertion of non-natural amino acids without requiring the resources of in vivo production," he said.
However, Boyle thinks it will be important to discern how widely applicable Giralt's method of cutting up the domain is. "It's not clear how many proteins or domains have noncovalent binding configurations that lend themselves to this approach," he said.

Giralt acknowledged that the screening of interactions between two separate sequences of the protein could produce results that are not representative of the interactions within the whole sequence. This underscores the importance of cutting the domain in a way that gives the screening test minimal potential for misleading results, he said.

Giralt and his group will next redesign proteins to incorporate both $\mathrm{L}$ - and $\mathrm{D}$-amino acids. $\mathrm{D}$-amino acids are resistant to proteases and can improve the pharmacokinetic properties of proteins containing them. "It is not easy to build a good ligand containing D-amino acids that is analogous to the natural ligand built from L-amino acids," Giralt said, "but our one-bead-one-compound approach to peptide synthesis can be a useful tool for doing so."

\section{REFERENCES}

1. Pastor, J. et al. Org. Lett. 22, 3831-3833 (2002)

2. Pastor, J. et al. J. Am. Chem. Soc.; published online Nov. 9, 2007; doi:10.1021/ja073969x

Contact: Ernest Giralt, Institute for Research in Biomedicine, Barcelona, Spain

e-mail: egiralt@pcb.ub.cat

\section{COMPANIES AND RESEARCH INSTITUTIONS MENTIONED Anaptys Biosciences Inc., La Jolla, Calif. Institute for Research in Biomedicine, Barcelona, Spain University of Barcelona, Barcelona, Spain Xencor Inc., Monrovia, Calif.}

